\title{
Sutureless closure: a versatile treatment for the diverse presentations of gastroschisis
}

\author{
David F. Grabski ${ }^{1}$ - Yinin $\mathrm{Hu}^{1}$ - Rick D. Vavolizza ${ }^{2}$ - Sara K. Rasmussen ${ }^{3}$ - Jonathan R. Swanson ${ }^{4}$. \\ Eugene D. McGahren ${ }^{3} \cdot$ Jeffrey W. Gander $\mathbb{D}^{3}$
}

Received: 14 September 2018 / Revised: 12 December 2018 / Accepted: 16 December 2018 / Published online: 28 January 2019

(c) Springer Nature America, Inc. 2019

\begin{abstract}
Objective Case series have demonstrated sutureless closures to be safe for the correction of gastroschisis. We hypothesize that sutureless closure is efficacious in patients requiring silo reduction without need for intubation.

Study design We conducted a retrospective case control study of infants who underwent gastroschisis repair at our institution (January 2011-August 2018). Patient characteristics and clinical outcomes were compared between sutureless closure and primary fascial repair groups.

Results Seventeen patients in the sutureless group and 28 patients in the primary fascial repair group were included. Success of sutureless closure was $94 \%$. Mechanical ventilation was reduced by 2.8 days in the sutureless group $(P<0.0001)$ and fewer patients required general anesthesia $(29.4 \%$ vs. $100 \%, P<0.0001)$.

Conclusions Sutureless closure is effective for the diverse presentations of gastroschisis. Given the concerns of effects of general anesthesia on the developing brain, sutureless closure should be strongly considered.
\end{abstract}

\section{Introduction}

Gastroschisis is a congenital defect of the anterior abdominal wall characterized by the absence of a membrane covering the herniated viscera. Gastroschisis occurs in $2-5$ of every 10,000 live births and is increasing in incidence [1, 2]. While the exact etiology of the disease is unknown, a vascular defect is suspected [3]. There is a correlation with other congenital and chromosomal anomalies and an increased incidence of intestinal injury leading to bowel ischemia and perforation $[4,5]$.

Jeffrey W. Gander

jg9br@virginia.edu

1 Department of Surgery, University of Virginia, Charlottesville, VA, USA

2 University of Virginia School of Medicine, Charlottesville, VA, USA

3 Division of Pediatric Surgery, University of Virginia, Charlottesville, VA, USA

4 Department of Pediatrics, University of Virginia, Charlottesville, VA, USA
Surgical management of gastroschisis has advanced over the last three decades [6], evolving from primary fascial repair under general anesthesia to a staged, silo-based reduction. Compared to early primary repair, a staged silo reduction allows for a more gradual increase in intraabdominal pressure and reduces ventilatory effects [7, 8]. More recently, a bedside reduction technique that culminated in a sutureless closure has become common [9]. With this method, the umbilical cord is intentionally left long and is used as a biologic dressing to cover the defect after reduction of abdominal contents. The sutureless repair has the same theoretical advantage of the staged silo reduction as the fascia remains open in the early postnatal period [9]. Case series report reduced need for mechanical ventilation and general anesthesia with sutureless repair as compared to primary fascial repair [10-12]. Experiences in staged reduction without general anesthesia have led some clinicians to pursue sutureless closure in the neonatal intensive care unit (NICU) with total circumvention of intubation and paralysis [13-16]. The sutureless approach also offers potential advantages in cosmetic results and hospital resource utilization [17-19].

Gastroschisis is a heterogenous congenital anomaly. The defect size, domain of the abdominal wall, as well as the presence of atresia or perforation vary amongst patients. 

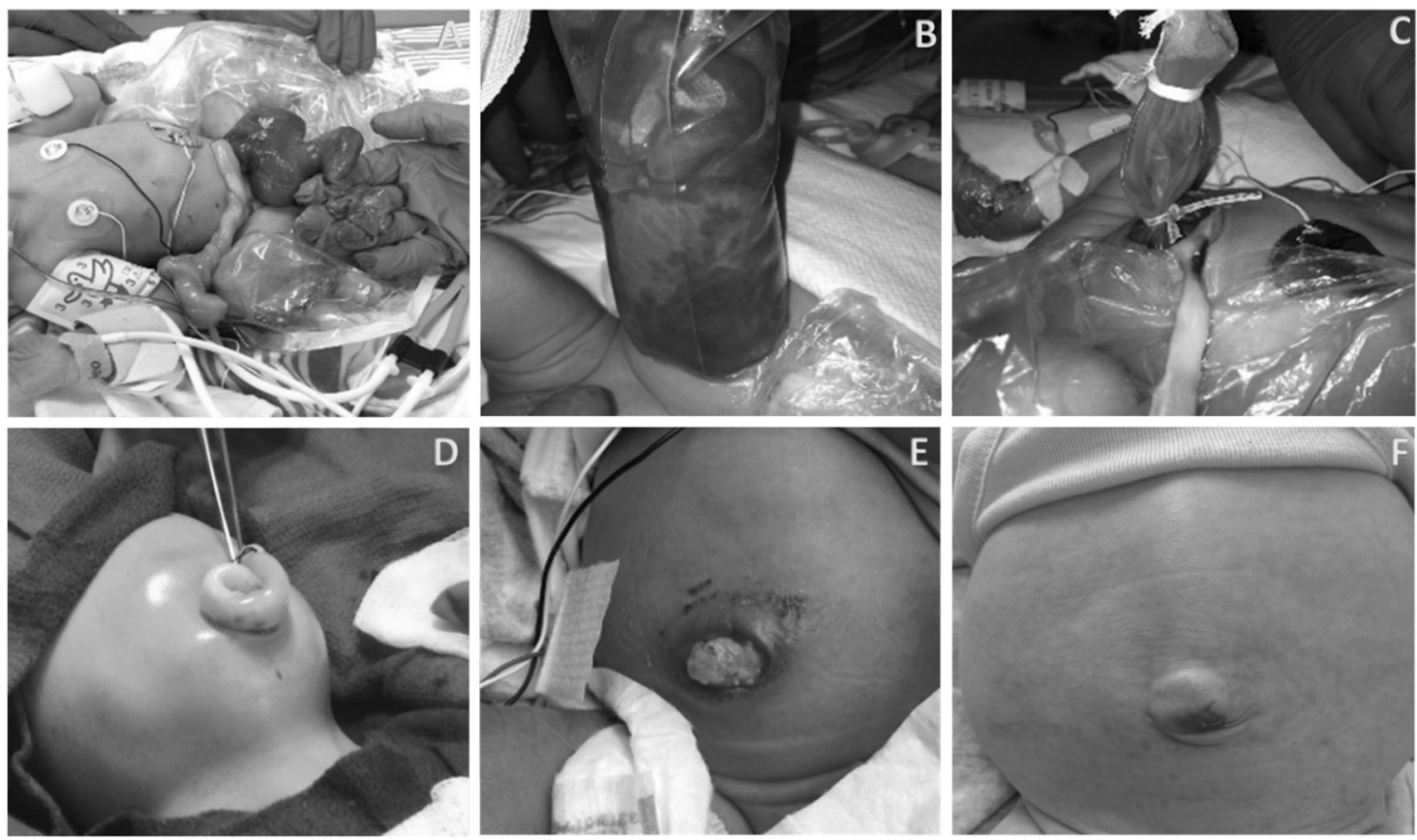

Fig. 1 Sutureless gastroschisis repair steps. a Identification of gastroschisis (this patient also has ileal atresia and necrotic bowel). b Placement of silo and protect umbilical cord. c Serial reductions until

Nearly all of the existing studies of sutureless closure exclude children born with complex gastroschisis (bowel ischemia, perforation, or atresia) [20]. There are also a limited number of studies that report on silo placement without intubation, followed by staged reduction and ultimate sutureless closure. The applications of sutureless closure in the diverse clinical presentations of gastroschisis have yet to be fully explored. We hypothesize that sutureless closure can be efficacious in patients requiring silo reduction without need for intubation. Additionally, the technique can be applied to patients with complex gastroschisis. We further hypothesize that sutureless repair will be associated with reductions in mechanical ventilation and general anesthesia as compared to primary fascial repair without compromising return of bowel function or length of stay.

\section{Methods}

After obtaining Institutional Review Board approval (UVA \#18450), we performed a retrospective case control study of infants who underwent gastroschisis repair at the University of Virginia Children's Hospital from January 2011 to August 2018. After August 2014, the sutureless closure technique was implemented at an increasing frequency based on primary surgeon preference. There were no other major medical, nutritional, or ventilatory management protocol changes in the neonatal intensive care unit over the bowel is below level of fascia. d Use of umbilical cord as biologic dressing. e Allow tissue to granulate (1 week). f Healed defect with umbilical hernia (6 months)

study period. Patients were identified utilizing a prospectively maintained clinical data repository derived from our institutional electronic medical record system. We included all neonates who underwent a gastroschisis repair, including neonates with silo use and complex gastroschisis. Patients were excluded if they transferred to another facility after the operation but prior to full recovery. We compared neonates who underwent a primary fascial closure with or without delayed silo reduction to those who underwent sutureless closure in an intention to treat analysis.

Sutureless closure was approached in a similar manner as described by Sandler et al. (Figs. 1 and 2) [9]. The obstetric team was instructed to leave the umbilical cord intentionally long $(\sim 30 \mathrm{~cm})$. After delivery, the neonate's eviscerated organs were placed in a bowel bag. Intubation was not performed for silo placement or full reduction unless the infant was in respiratory distress as deemed by the clinical team. Intravenous access was obtained and the patient received standard doses of fentanyl $(1 \mathrm{mcg} / \mathrm{kg})$ and midazolam $(0.1 \mathrm{mg} / \mathrm{kg})$. Under sterile conditions, the exposed bowel was assessed for obvious intestinal pathology by the surgical team. The surgical team then determined whether the bowel could be completely reduced below the level of the fascia without affecting the ventilation. If this was not possible, a spring-loaded silo was applied for staged reduction. The umbilical cord was wrapped in a moist gauze and plastic wrap. The bowel was then gently reduced in the silo daily while the nursing staff kept the umbilical cord moist. For silo placement and removal, pain medication was 
Fig. 2 Flow diagram of sutureless closure algorithm including critical steps

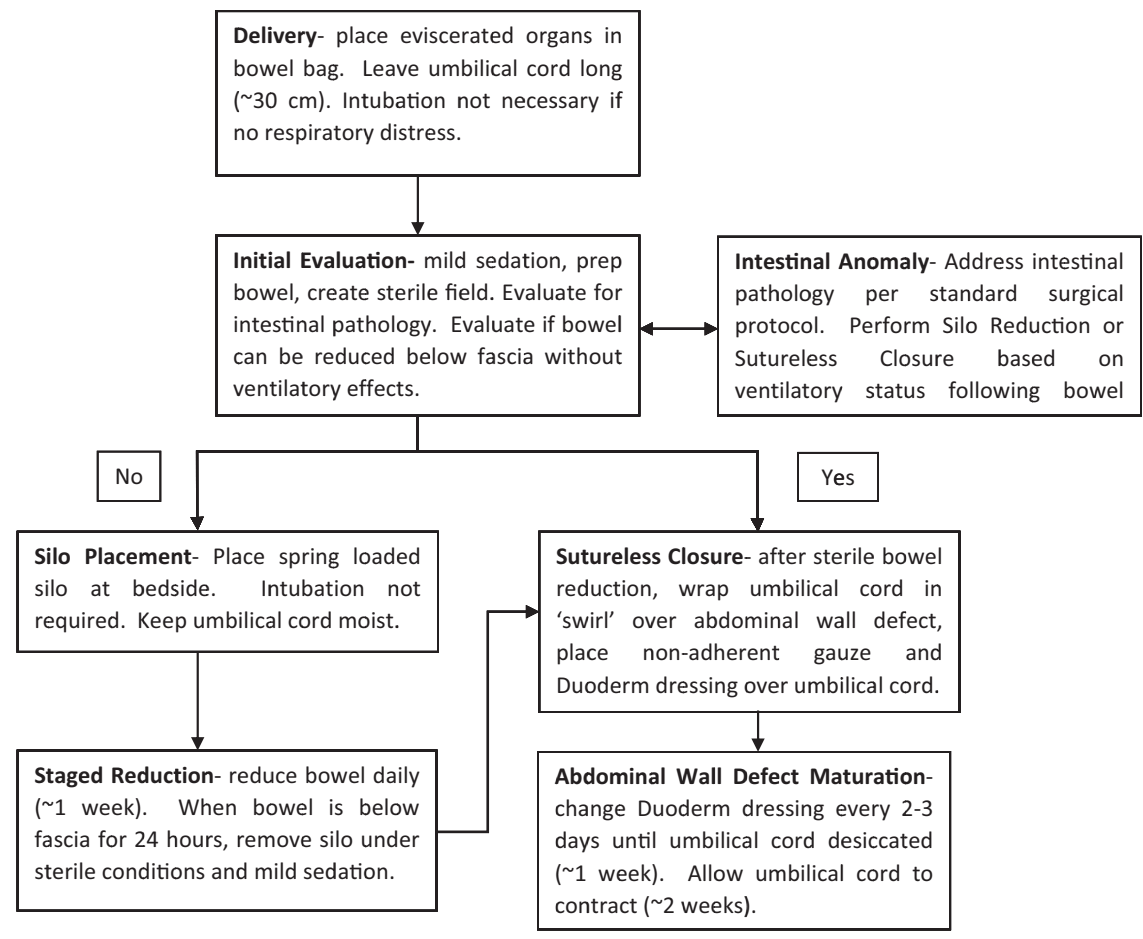

given (Fentanyl $1 \mathrm{mcg} / \mathrm{kg}$ ). Tylenol was utilized for discomfort, but opioid infusion was generally not required while the silo was in place. After the bowel was below the fascia for $24 \mathrm{~h}$, sutureless closure was performed. A sterile environment was created, the patient was given $1 \mathrm{mcg} / \mathrm{kg}$ of fentanyl and the silo removed. The umbilical cord was cleaned with betadine and placed in a "swirl" configuration over the defect. It was dressed with a non-adherent gauze and Duoderm dressing (ConvaTec, Deeside, United Kingdom). This was changed every 2-3 days until the umbilical cord was adherent and began to desiccate. The umbilicus was then allowed to contract while monitoring for signs of infection. This usually takes $\sim 2-3$ weeks.

Neonates who were born with an additional intestinal anomaly (necrotic intestine or perforation of intestinal tract) were taken to the operating room or had a bedside procedure to manage the emergent condition. The bowel was placed in a silo for later reduction. The umbilical cord was protected for use as a sutureless closure technique as described above. Neonates with intestinal atresia were managed with a sutureless closure followed by exploration 7 weeks later for anastomosis.

Patients in the primary fascial repair group were managed similarly in determining whether the child could be completely reduced or would require silo placement. They would later undergo fascial closure in the operating room under general anesthesia using standard techniques.

Clinical data were collected on all patients who met inclusion criteria. Collected data included demographic and birth history, operative details, mechanical ventilation days, days to full enteral nutrition (defined as discontinuation of total parenteral nutrition), use of general anesthesia, use of the operating room, length of hospitalization, surgical site infection, and in-hospital mortality. The primary clinical outcomes were mechanical ventilation days, and general anesthesia use. Secondary outcomes included time to full enteral feeds, length of stay, and mortality. A subgroup analysis was conducted for patients with complex gastroschisis as a feasibility analysis.

Clinical outcomes were summarized using mean and standard deviation for normal data and median and interquartile range (IQR) for non-normal data. The Student's $t$ test, the Wilcoxon Rank Sum Test and Fisher's Exact Test were used as appropriate. All hypothesis tests were considered two-sided and a p-value of 0.01 was considered significant to account for our multi-outcome analysis and reduce potential bias. All analyses were conducted in SAS Version 9.4 (Cary, NC, USA).

\section{Results}

Forty-six neonates underwent gastroschisis repair over the study period. One infant was transferred to another facility after fascial repair due to geographic convenience. In total, 45 infants met inclusion criteria; 28 in the primary fascial repair group and 17 in the sutureless closure group. Sixteen of the 17 patients (94\%) who underwent the sutureless closure technique were successfully treated without conversion to the standard fascial closure technique. Early in 
Table 1 Patient characteristics for sutureless and fascial repairs

\begin{tabular}{llll}
\hline Patient characteristics & $\begin{array}{l}\text { Sutureless } \\
\text { closure }\end{array}$ & $\begin{array}{l}\text { Fascial } \\
\text { closure }\end{array}$ & Significance \\
\hline $\begin{array}{l}\text { Total patients } \\
\text { Maternal age at } \\
\text { delivery (years) }\end{array}$ & $23.6(4.9)$ & $22.5(3.7)$ & $P=0.387$ \\
$\begin{array}{l}\text { Cesarean delivery } \\
\text { Maternal gestational }\end{array}$ & $7(38.9 \%)$ & $10(37.0 \%)$ & $P=0.900$ \\
comorbidities & 0 & 1 & $P=0.431$ \\
$\begin{array}{l}\text { Gender (male) } \\
\begin{array}{l}\text { Gestational age } \\
\text { (weeks) }\end{array}\end{array}$ & $9(53 \%)$ & $8(29 \%)$ & $P=0.124$ \\
$\begin{array}{l}\text { Birth weight (kg) } \\
\text { APGAR }\end{array}$ & $2.36(0.36)$ & $2.52(0.59)$ & $P=0.311$ \\
$\quad$ 1 min & $8(7,8)$ & $8(3,8)$ & $P=0.575$ \\
$\quad 5$ min & $9(8,9)$ & $8(7,9)$ & $P=0.038$ \\
Complex gastroschisis & 3 & 3 & $P=0.658$ \\
\hline
\end{tabular}

Complex gastroschisis-bowel ischemia, perforation, or atresia. Maternal gestational comorbidities-pre-eclampsia, gestational diabetes, complication at birth. Central tendency is described by mean and standard deviation or median and inter-quartile range

our experience with sutureless closure, one child eviscerated their intestine when the umbilical cord was placed over the defect. They subsequently were intubated, taken to the operating room, and underwent a standard fascial repair. This patient was analyzed in the sutureless repair group. The number of patients with complex gastroschisis was the same in both groups $(n=3)$.

Patient characteristics were similar between the groups and are summarized in Table 1. The maternal age, delivery type, and maternal gestational comorbidities were similar across groups. The mean gestational age was $36.8( \pm 2.03)$ weeks in the sutureless closure group and $36.7( \pm 1.31)$ weeks in the primary fascial repair group $(P=0.90)$. The average birth weight was similarly distributed between the two groups with the sutureless closure group weighing 2.36 $( \pm 0.36) \mathrm{kg}$ and the primary fascial repair group weighing $2.52(0.59) \mathrm{kg}(P=0.32)$.

Clinical outcomes are summarized in Table 2. Sutureless closure was associated with significantly shorter duration of mechanical ventilation compared to primary fascial repair $(0 \mathrm{~h}(0,25)$ vs. $68 \mathrm{~h}[53,113] ; P<0.0001)$. Intubation was never required in $9 / 17(52.9 \%)$ patients in the sutureless closure group. When patients with complex gastroschisis who required intubation for bowel repair are excluded from the sutureless closure group, 9/14 (64.2\%) patients were managed without intubation. Conversely, 28/28 (100\%) of patients in the primary fascial group required intubation $(P$ $<0.0001)$. All patients with complex gastroschisis, regardless of repair type were intubated for their initial emergency surgery. Of the patients who were intubated in this group, three were intubated at birth. One was for respiratory distress, one for transport from an outside institution and the other due to the need to perform lysis of adhesions in the operating room as the bowel was matted to the abdominal wall skin. The other two patients required intubation secondary to bowel evisceration at time of final sutureless closure. One was converted to standard fascial closure and the other was successfully closed with the sutureless technique at bedside. That patient was extubated the following morning.

The use of general anesthesia over the initial hospitalization was also statistically reduced in the sutureless closure group $(29.4 \%$ vs. $100 \%, P<0.0001)$, as was operating room usage $(29.4 \%$ vs. $100 \%, P<0.0001)$. Both groups required similar use of silos, $88 \%$ in sutureless closure and $75 \%$ in primary fascial closure, with a median of 6 days (IQR 4-8) in the sutureless closure group and 8 days (IQR $2.5-10)$ in the fascial repair group $(P=0.311)$. Of note, in the sutureless closure group, three infants had silos in place for 10 days prior to sutureless closure and all of these cases were successful.

Both repair groups had similar times to full enteral feeds, with infants in the sutureless closure group requiring 28 days for bowel recovery and children in the primary fascial group requiring 29 days $(P=0.347)$. The average length of hospitalization 31 days (IQR 27, 57 days) with the sutureless closure and 36 days (IQR 27, 39 days) in the primary fascial repair group $(P=0.423)$. There was one surgical site infection in the sutureless closure group and three in the primary fascial repair group $(P=0.863)$. A total of four infants treated for gastroschisis died over the study period for an overall 1-year mortality of $8.8 \%$. Three deaths were in the primary fascial repair group. Two patients died from sequela of lethal genetic anomalies and one died from respiratory cytomegalovirus infection and resulting septic shock. One patient in the sutureless repair group died from a central line complication after initial hospitalization discharge. The patient was born with ileal atresia and required a significant bowel resection resulting in short-gut syndrome and TPN dependence.

There were three patients with complex gastroschisis in each group (clinical outcomes of complex gastroschisis summarized in Table 3). Given the small number of patients, statistical analysis was not conducted. In the sutureless closure group, two children were born with intestinal atresia and one with a gastric perforation. They required an average of 5.5 days for silo-based intestinal reduction. Both patients with an intestinal atresia had obvious necrotic bowel and required bowel resection prior to silo placement and eventual sutureless closure. Both patients with ileal atresia returned to the operating room 7 weeks after sutureless closure for atresia repair, which is the standard repair interval for concomitant ileal atresia and gastroschisis. The patient with the gastric perforation was 
Table 2 Clinical outcomes for sutureless and fascial repairs

\begin{tabular}{lccc}
\hline Clinical characteristics & Sutureless repair $(n=17)$ & Fascial repair $(n=28)$ & Significance \\
\hline Patients placed in silo & $15(88 \%)$ & $21(75 \%)$ & $P=0.447$ \\
Days in silo & $6(4,8)$ & $8(2.5,10)$ & $P=0.209$ \\
Time to 100\% enteral deeds (days) & $28(25,48)$ & $29(23,34)$ & $P=0.265$ \\
Patients intubated & $8(47 \%)$ & $28(100 \%)$ & $P<0.0001^{*}$ \\
Cumulative hours on ventilator & $0(0,25)$ & $68.0(53,113)$ & $P<0.0001^{*}$ \\
Patients requiring general anesthesia & $5(29.4 \%)$ & $28(100 \%)$ & $P<0.0001^{*}$ \\
Patients requiring operating room & $5(29.4 \%)$ & $28(100 \%)$ & $P<0.0001^{*}$ \\
Length of hospitalization (days) & $31(27,57)$ & $36(27,39)$ & $P=0.423$ \\
Infection & $1(5.8 \%)$ & $3(10.7 \%)$ & $P=0.863$ \\
Mortality (1 year) & $1(5.8 \%)$ & $3(10.7 \%)$ & $P=0.863$ \\
\hline
\end{tabular}

Central tendency is described by mean and standard deviation or median and inter-quartile range noted after delivery. The cause could not be determined. The patient was taken to the operating room for closure of the perforation and silo placement. A sutureless closure was performed after serial reduction of the bowel. There were no major complications associated with the sutureless closure for complex gastroschisis.

\section{Discussion}

Sutureless closure is a versatile treatment for the diverse presentations of gastroschisis. In this series, sutureless closure was successful in $94 \%$ of patients, including three patients with complex gastroschisis. The use of sutureless closure has previously been reserved for patients who were able to be reduced primarily and not require a silo [20]. This practice has evolved as some groups now use sutureless closure if a silo needs to be placed initially [16, 21]. Investigations have further showed that sutureless closure can be applied even if the silo is used for a prolonged period of time. In a randomized control trial of sutureless closure and primary fascial repair, Bruzoni et al. had a mean length of silo use of 9 days [21]. In our series the length of silo use was 6 days. However, in most series, the patient is intubated for bedside silo placement. The present study is unique because intubation for patients for silo placement was not required unless there was significant pre-procedural respiratory distress. This protocol led to reduced length of mechanical ventilation as the silo could be removed and sutureless closure performed at the bedside without intubation.

Riboh et al. noted a significant reduction in length of ventilation from 12.1 days in the fascial closure group to 5.0 in the sutureless group [10]. When the same institution later conducted a randomized trial evaluating the different repair techniques, Bruzoni and colleagues noted that the sutureless approach required 1.89 days of mechanical ventilation, while the sutured group required 3.15 days. This result did not reach statistical significance, which the authors attributed to under powering [21]. In a separate series, Orioin et al. noted a 4-day reduction in ventilator requirement in the sutureless group [11]. Witt and colleagues similarly found that in a cohort of 90 patients, the sutureless closure technique reduced ventilatory requirements by 4 days [22]. In our study, the average length of intubation was significantly decreased by 2.8 days with the sutureless technique.

Recently, Pet el al. described a $65 \%$ success rate when performing sutureless closure in the NICU without initial intubation [16]. In our series, 52\% did not require intubation. When complex gastroschisis is excluded, $64 \%$ of our patients never required intubation. Reducing intubation length is clinically important as these patients will experience less barotrauma and potentially reduce the risk for chronic lung disease and pulmonary inflammation.

Most series exclude patients with complex gastroschisis for sutureless closure. However, in practice $10 \%$ of all patients with gastroschisis will be considered complex often due to intestinal atresia. In our series, the sutureless technique is beneficial for patients with atresia. Traditionally, a patient with an atresia and gastroschisis is managed with reduction of bowel with fascial closure. Four to eight weeks later they are taken to the operating room for repair of the atresia [23]. This requires multiple anesthetics and at least two visits to the operating room. The sutureless closure technique can avoid the subsequent anesthetic and intubation required for a fascial closure in patients with atresia as well. In the present study, two patients had an intestinal atresia and were treated with the sutureless technique. The first patient was early in our experience and was taken to the operating room for stapled resection of the necrotic atretic segment. They were later taken to the operating room for definitive repair of the atresia. The other patient only required an anesthetic for the atresia repair.

We found a significant reduction in the use of general anesthesia between the two groups. The sutureless closure 
Table 3 Clinical outcomes of complex gastroschisis in sutureless repair and fascial repair with sutureless non-complex cases for comparison

\begin{tabular}{llll}
\hline Clinical characteristics & $\begin{array}{l}\text { Sutureless repair in complex } \\
\text { gastroschisis }(n=3)\end{array}$ & $\begin{array}{l}\text { Fascial repair in complex } \\
\text { gastroschisis }(n=3)\end{array}$ & $\begin{array}{l}\text { Sutureless repair in non-complex } \\
\text { gastroschisis }(n=14)\end{array}$ \\
\hline $\begin{array}{l}\text { Patients placed in silo } \\
\text { Days in silo }\end{array}$ & $3(100 \%)$ & $3(100 \%)$ & $12(85.7 \%)$ \\
$\begin{array}{l}\text { Time to } 100 \% \text { enteral feeds } \\
\text { (days) }\end{array}$ & $5.53(1.15)$ & $14.3(7.09)$ & $6.08(3.52)$ \\
$\begin{array}{l}\text { Patients intubated } \\
\text { Cumulative hours on ventilator }\end{array}$ & $35(16,123)$ & $86(19,88)$ & $28(24,47)$ \\
$\begin{array}{l}\text { Patients requiring general } \\
\text { anesthesia }\end{array}$ & $3(100 \%)$ & $3(100 \%)$ & $5(35.7 \%)$ \\
$\begin{array}{l}\text { Patients requiring operating } \\
\text { room }\end{array}$ & $3(100 \%)$ & $167(104,960)$ & $3(23.1 \%)$ \\
$\begin{array}{l}\text { Length of hospitalization } \\
\text { days) }\end{array}$ & $94(29,123)$ & $3(100 \%)$ & $3(23.1 \%)$ \\
$\begin{array}{l}\text { Infection } \\
\text { Mortality (1 year) }\end{array}$ & 0 & $3(100 \%)$ & $30.5(27,55)$ \\
\hline
\end{tabular}

Complex gastroschisis-born with intestinal anomaly including atresia or perforation. No statistical analysis was completed given small number of complex gastroschisis cases

required general anesthesia in only $29.4 \%$ of patients while $100 \%$ of patients in primary fascial group required an anesthetic. There is growing concern for repeated anesthetics on the developing brain and reduced school performance [24]. A potential future benefit of the sutureless closure may improve neurodevelopment.

Most retrospective investigations demonstrate equivalent time to enteral feeding and length of stay between sutureless and primary repair techniques $[10,11,20]$. Our series also supports these findings. However, Bruzoni et al.'s recent randomized trial reported a significant increase in time to full feeds (45.1 vs. 27.8 days) and time to discharge (49.3 vs. 31.4 days) in the sutureless group compared to the standard fascial repair group [21]. The investigators of the study suggest several reasons why this may be including a prolonged silo reduction period (average 9 days in the study) leading to larger abdominal wall defects which take longer to close by secondary intention.

Several studies have noted significant reduction in surgical infection rates with sutureless closure [21, 25], which was a trend seen in our study as well. Previous reports indicate that sutureless closure technique may be less costly given the reduced need for operative resources, while billing and reimbursement otherwise remain the same between procedures $[18,19]$. We did not evaluate cost data in our study. However, in our study we reduced the usage of the operating room. Theoretically, this supports reduced total hospital costs in the sutureless repair and makes the operating room available for other patients in a timelier manner. University of Virginia Children's Hospital is a pediatric center within a large adult hospital. The majority of other sutureless studies have been performed in a stand-alone children's hospital. We feel that with little additional resources, the sutureless closure can be performed at all facilities that care for neonates.

As a single institution case control study, we could not control for confounding variables which may introduce selection bias. By specifically including patients with complex gastroschisis, we introduced a level of patient heterogeneity into our data. We did this to establish feasibility of the sutureless technique within this patient population, and to more closely approximate the reality of the gastroschisis population. We concentrated on short term, inhospital outcomes in this investigation. Long-term outcomes of sutureless closure including umbilical hernia repair rates were not possible given the length of follow up in our study.

\section{Conclusion}

This investigation supports the use of a bedside sutureless closure technique without mandatory intubation for repair of simple and complex gastroschisis. It reduces intubation length and need for general anesthetics. The sutureless repair strategy can be effective in a non-standalone pediatric hospital setting. These findings promote adoption of sutureless repair in all tertiary care facilities that treat this disease.

\section{Compliance with ethical standards}

Conflict of interest The authors declare that they have no conflict of interest. 
Publisher's note: Springer Nature remains neutral with regard to jurisdictional claims in published maps and institutional affiliations.

\section{References}

1. Wittekindt B, Schloesser R, Doberschuetz N, Salzmann-Manrique E, Grossmann J, Misselwitz B, et al. Epidemiology and outcome of major congenital malformations in a large German County. Eur J Pediatr Surg=Z. Kinderchir. 2018. E-pub ahead of print.

2. Jones AM, Isenburg J, Salemi JL, Arnold KE, Mai CT, Aggarwal D, et al. Increasing prevalence of gastroschisis-14 States, 19952012. Morb Mortal Wkly Rep. 2016;65:23-6.

3. Hoyme HE, Jones MC, Jones KL. Gastroschisis: abdominal wall disruption secondary to early gestational interruption of the omphalomesenteric artery. Semin Perinatol. 1983;7:294-8.

4. Stoll C, Alembik Y, Dott B, Roth MP. Omphalocele and gastroschisis and associated malformations. Am J Med Genet A. 2008;146a:1280-5.

5. Mastroiacovo P, Lisi A, Castilla EE, Martinez-Frias ML, Bermejo E, Marengo L, et al. Gastroschisis and associated defects: an international study. Am J Med Genet A. 2007;143a:660-71.

6. Chesley PM, Ledbetter DJ, Meehan JJ, Oron AP, Javid PJ. Contemporary trends in the use of primary repair for gastroschisis in surgical infants. Am J Surg. 2015;209:901-5. discussion5-6

7. Fischer JD, Chun K, Moores DC, Andrews HG. Gastroschisis: a simple technique for staged silo closure. J Pediatr Surg. 1995;30:1169-71.

8. Minkes RK, Langer JC, Mazziotti MV, Skinner MA, Foglia RP. Routine insertion of a silastic spring-loaded silo for infants with gastroschisis. J Pediatr Surg. 2000;35:843-6.

9. Sandler A, Lawrence J, Meehan J, Phearman L, Soper R. A "plastic" sutureless abdominal wall closure in gastroschisis. J Pediatr Surg. 2004;39:738-41.

10. Riboh J, Abrajano CT, Garber K, Hartman G, Butler MA, Albanese CT, et al. Outcomes of sutureless gastroschisis closure. J Pediatr Surg. 2009;44:1947-51.

11. Orion KC, Krein M, Liao J, Shaaban AF, Pitcher GJ, Shilyansky J. Outcomes of plastic closure in gastroschisis. Surgery. 2011;150:177-85.

12. Zalles-Vidal C, Penarrieta-Daher A, Bracho-Blanchet E, IbarraRios D, Davila-Perez R, Villegas-Silva R, et al. A Gastroschisis bundle: effects of a quality improvement protocol on morbidity and mortality. J Pediatr Surg. 2018;53:2117-22.
13. Bianchi A, Dickson AP. Elective delayed reduction and no anesthesia: 'minimal intervention management' for gastrochisis. J Pediatr Surg. 1998;33:1338-40.

14. Kimble RM, Singh SJ, Bourke C, Cass DT. Gastroschisis reduction under analgesia in the neonatal unit. J Pediatr Surg. 2001;36:1672-4.

15. Davies MW, Kimble RM, Cartwright DW. Gastroschisis: ward reduction compared with traditional reduction under general anesthesia. J Pediatr Surg. 2005;40:523-7.

16. Pet GE, Stark RA, Meehan JJ, Javid PJ. Outcomes of bedside sutureless umbilical closure without endotracheal intubation for gastroschisis repair in surgical infants. Am J Surg. 2017; 213:958-62.

17. Zajac A, Bogusz B, Soltysiak P, Tomasik P, Wolnicki M, Wedrychowicz A, et al. Cosmetic outcomes of sutureless closure in gastroschisis. Eur J Pediatr Surg=Z Kinderchir. 2016;26:537-41.

18. Cauchi J, Parikh DH, Samuel M, Gornall P. Does gastroschisis reduction require general anesthesia? A comparative analysis. J Pediatr Surg. 2006;41:1294-7.

19. Wu JX, Lee SL, DeUgarte DA. Cost modeling for management strategies of uncomplicated gastroschisis. J Surg Res. 2016;205:13641.

20. Choi WW, McBride CA, Bourke C, Borzi P, Choo K, Walker R, et al. Long-term review of sutureless ward reduction in neonates with gastroschisis in the neonatal unit. J Pediatr Surg. 2012;47:1516-20.

21. Bruzoni M, Jaramillo JD, Dunlap JL, Abrajano C, Stack SW, Hintz SR, et al. Sutureless vs sutured gastroschisis closure: a prospective randomized controlled trial. J Am Coll Surg. 2017;224:1091-6.e1.

22. Witt RG, Zobel M, Padilla B, Lee H, MacKenzie TC, Vu L. Evaluation of clinical outcomes of sutureless vs sutured closure techniques in gastroschisis repair. JAMA Surg. 2018. E-pub ahead of print.

23. Snyder CW, Biggio JR, Brinson P, Barnes LA, Bartle DT, Georgeson KE, et al. Effects of multidisciplinary prenatal care and delivery mode on gastroschisis outcomes. J Pediatr Surg. 2011;46:86-9.

24. Schneuer FJ, Bentley JP, Davidson AJ, Holland AJ, Badawi N, Martin AJ, et al. The impact of general anesthesia on child development and school performance: a population-based study. Paediatr Anaesth. 2018;28:528-36.

25. Schlueter RK, Azarow KS, Hines AG, Varman M, Abdessalam $\mathrm{SF}$, Raynor SC, et al. Identifying strategies to decrease infectious complications of gastroschisis repair. J Pediatr Surg. 2015; 50:98-101. 